\title{
Efficacy of Low-Level Laser Therapy in the Management of Tinnitus due to Noise-Induced Hearing Loss: A Double-Blind Randomized Clinical Trial
}

\author{
Abolfazl Mollasadeghi, ${ }^{1}$ Seyyed Jalil Mirmohammadi, ${ }^{1}$ \\ Amir Houshang Mehrparvar, ${ }^{1}$ Mohammad Hossein Davari, ${ }^{1}$ Pedram Shokouh, ${ }^{2}$ \\ Mehrdad Mostaghaci, ${ }^{1}$ Mohammad Hossein Baradaranfar, ${ }^{3}$ and Maryam Bahaloo, ${ }^{4,5}$ \\ ${ }^{1}$ Department of Occupational Medicine, School of Medicine, Shahid Sadoughi University of Medical Sciences, \\ P.O. Box 89138-14389, Yazd, Iran \\ ${ }^{2}$ Cardiovascular Research Center, Isfahan Cardiovascular Research Institute, Isfahan University of Medical Sciences, \\ P.O. Box 81465-1148, Isfahan, Iran \\ ${ }^{3}$ Department of Otolaryngology and Head and Neck Surgery, School of Medicine, Yazd University of Medical Sciences, \\ P.O. Box 89138-14389, Yazd, Iran \\ ${ }^{4}$ Industrial Diseases Research Center, School of Medicine, Shahid Sadoughi University of Medical Sciences, \\ P.O. Box 89138-14389, Yazd, Iran \\ ${ }^{5}$ Shahid Rahnamoun Hospital, Farrokhi Ave, Yazd, Iran
}

Correspondence should be addressed to Maryam Bahaloo; drbahaloo@gmail.com

Received 1 June 2013; Accepted 12 September 2013

Academic Editors: C. Cingi and F. Oghan

Copyright (C) 2013 Abolfazl Mollasadeghi et al. This is an open access article distributed under the Creative Commons Attribution License, which permits unrestricted use, distribution, and reproduction in any medium, provided the original work is properly cited.

Background. Several remedial modalities for the treatment of tinnitus have been proposed, but an effective standard treatment is still to be confirmed. In the present study, we aimed to evaluate the effect of low-level laser therapy on tinnitus accompanied by noiseinduced hearing loss. Methods. This was a double-blind randomized clinical trial on subjects suffering from tinnitus accompanied by noise-induced hearing loss. The study intervention was 20 sessions of low-level laser therapy every other day, 20 minutes each session. Tinnitus was assessed by three methods (visual analog scale, tinnitus handicap inventory, and tinnitus loudness) at baseline, immediately and 3 months after the intervention. Results. All subjects were male workers with age range of 30-51 years. The mean tinnitus duration was $1.85 \pm 0.78$ years. All three measurement methods have shown improved values after laser therapy compared with the placebo both immediately and 3 months after treatment. Laser therapy revealed a U-shaped efficacy throughout the course of follow-up. Nonresponse rate of the intervention was $57 \%$ and $70 \%$ in the two assessment time points, respectively. Conclusion. This study found low-level laser therapy to be effective in alleviating tinnitus in patients with noise-induced hearing loss, although this effect has faded after 3 months of follow-up. This trial is registered with the Australian New Zealand clinical trials registry with identifier ACTRN12612000455864.

\section{Introduction}

Tinnitus is defined as a sound in the ear(s) without any external auditory stimulus. About $15 \%$ of the general population experience at least one episode of tinnitus, which prevalence increases by age and reaches $85 \%$ in individuals older than 60 years [1]. This symptom is intolerable in nearly $20 \%$ of the cases [2]. Reaching as high as $67 \%$, tinnitus is more prevalent among individuals suffering from hearing disorders [3].
Noise has such deleterious effects on hearing as noiseinduced hearing loss (NIHL) is the second most common form of acquired hearing loss. It has long been recognized as a problem in noisy environments workers [4]. As a possible complication of NIHL, tinnitus is usually observed at frequencies equal to or higher than $3000 \mathrm{~Hz}$, which is one octave band higher than the frequencies affected in NIHL. Its intensity is usually between 3 and $5 \mathrm{~dB}$ (occasionally up to $15 \mathrm{~dB}$ ) [5]. 
Tinnitus may lead to such complications as depression, irritability, sleep disorders, and loss of concentration [6]. Although lacking a widely accepted treatment, various therapeutic modalities have been proposed thus far, including medications (such as sedatives, antiepileptics, antidepressants, antipsychotics, local anesthetics, antihistamines, and botulinum toxin A) [7], repetitive transcranial magnetic stimulation [8], transcutaneous electrical stimulation [9], and sound therapy [10]. Low-level laser therapy (LLLT) has recently been tried with promising results in outpatients with subjective tinnitus [2].

As known, laser has different usages in medicine such as wound healing, nerve and tissue repairing, pain control [11], and treating Meniere's disease and tinnitus [12]. Although the exact mechanism of the effect of LLLT on tinnitus is not clearly understood, it has been proposed that it may be induced by increasing cell proliferation, growth factor secretion, improvement in inner ear blood flow, and/or activation of the hair cells mitochondria [2]. There is still some degree of controversy concerning the efficiency of LLLT in tinnitus. Some studies have shown positive effects $[2,11,13$, $14]$, but others have found no such effectiveness $[15,16]$.

Considering the fact that NIHL is a common disorder in industrial settings and tinnitus is its most common associated subjective complaint, we designed an interventional study to evaluate the effect of LLLT on tinnitus accompanied by NIHL.

\section{Methods}

2.1. Study Design and Population. The present study was a double-blind randomized clinical trial with the participation of patients referred to the occupational medicine clinic of Shahid Sadoughi University of Medical Sciences. Recruitment took place from September 2010 till September 2011.

One hundred volunteers younger than 50 years suffering from NIHL (defined as a bilateral sensorineural hearing loss, with the hearing threshold higher than $15 \mathrm{~dB}$ at least at one of the following frequencies: 3000,4000 , and $6000 \mathrm{~Hz}$ [4]) and tinnitus have enrolled to the study. The level of effect observed in a former study was used for the calculation of the sample size [2].

After baseline screening interview and examination, eleven participants were excluded from the study, yielding a final sample size of 89 . Our main exclusion criteria were as follows: any history of exposure to ototoxic drugs/substances, psychotic disorders with auditory hallucination, acoustic trauma, head trauma, mumps, meningitis, Meniere's disease, and having any contraindication for laser therapy [17].

Subjects were randomly allocated to either laser therapy or placebo groups. Randomization was done using a random digit table. According to the principles of double blindness, the study participants and operators who performed the assessment tests as well as the researchers who evaluated the outcomes were completely blinded to the groups.

After taking a thorough medical and occupational history, the microscopic examination of auditory meatus and tympanic membrane was performed. Afterwards, subjects underwent pure-tone audiometry performed at 250, 500, $1000,2000,3000,4000,6000$, and $8000 \mathrm{~Hz}$ frequencies (device: clinical audiometer, Interacoustic, AC40; headphone: TDH39, Denmark) in an acoustic chamber meeting the American National Standards Institute criterions [18]. Tympanometry was also accomplished for all participants (device: Tympanometer, Interacoustic, AZ26, Denmark). Subjects in the intervention group underwent laser therapy for 20 sessions, every other day, 20 minutes each session, which was a combination of protocols used in the previous studies $[1,2,13]$. A low-level laser beam with wave length of $650 \mathrm{~nm}$ and intensity of $5 \mathrm{~mW}$ was irradiated to the ear via mastoid bone (device: TINNImed, Switzerland). This device was connected to the ear by a soft silicone tip. The treatment sessions were performed for the subjects in placebo group with turned-off device.

A written informed consent was obtained from all participants before the enrolment. The protocol of the study was approved by the ethics committee of research vice chancellor of Shahid Sadoughi University of Medical Sciences.

2.2. Efficacy Assessments. We used the following three validated methods for the evaluation of outcome before treatment, immediately and 3 months after the termination of treatment: tinnitus visual analog scaling (VAS), tinnitus handicap inventory (THI), and tinnitus loudness measurement. Visual analog scale is scored on a 10 -point scale, in which individuals select the lowest perceived loudness on a scale of 0 to 10 corresponding to an increasing level of loudness [19]. In THI scoring, 25 questions are asked from the patient and the severity of tinnitus is categorized as follows. Grade 1 (0-16): Slight (only heard in quiet environments); Grade 2 (18-36): Mild (easily masked by environmental sounds and easily forgotten with activities); Grade 3 (3856): Moderate (noticed in the presence of background noise, although daily activities can still be performed); Grade 4 (5876): Severe (almost always heard, leads to disturbed sleep patterns and can interfere with daily activities); Grade 5 (78100): Catastrophic (Always heard, disturbed sleep patterns, difficulty with any activities) [20]. We used a translated version of the questionnaire into Persian, which was reviewed and modified by three experts to adapt our population culture. Loudness and frequency of tinnitus was assessed by audiometer. Pitch was matched by introducing two successive tones to the ear and the patient chose which one was closest to the tinnitus pitch. The loudness was assessed by matching it with the loudness of pure tone at each frequency in the contralateral ear according to the patient's sensation.

\subsection{Statistical Analysis. Data were analyzed by the Statistical} Package for Social Sciences software version 15.0 (SPSS Inc, Chicago, Illinois, USA). We used independent-sample $t$ test for the comparison of mean tinnitus loudness between two groups in three occasions (baseline, immediately, and 3 months after intervention), and paired $t$-test for the comparison of treatment effect within each group in different occasions. Chi square test was also employed in the comparison of VAS and THI score changes between two groups. 


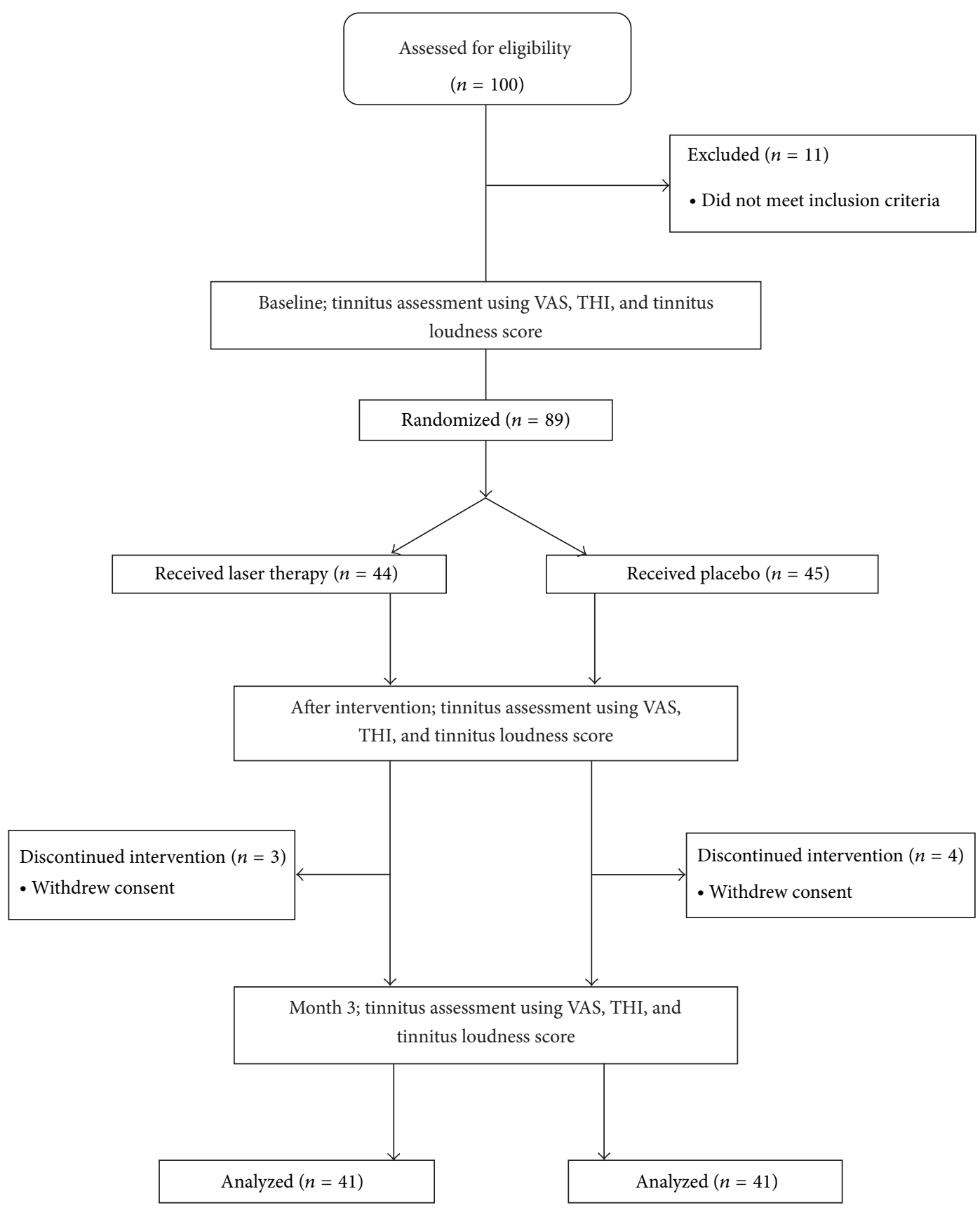

Figure 1: Study flow diagram.

\section{Results}

From 100 patients screened, 89 individuals were eligible for enrolment. Reasons for exclusion were as follows: exposure to ototoxic substances $(n=6)$, head injury $(n=2)$, consumption of ototoxic drug $(n=1)$, head trauma $(n=1)$, and childhood infection $(n=1)$. Figure 1 shows the flow diagram of the study. As demonstrated, 3 laser therapy- and 4 placebo-assigned participants have discontinued the trial due to personal reasons. Notably, no case of LLLT-attributable side effects was observed in our course of study.

All cases were males with age range of 30 to 51 years (mean: $41.17 \pm 5.89$ years). Their mean duration of employment was $12.21 \pm 1.77$ years. Mean level of noise in the workplace (time weighted average for an 8-hour shift) was $87.60 \pm 1.49 \mathrm{dBA}$. Tinnitus was bilateral in $49 \%$ of the cases, while 27 and 24 percent of subjects suffered from unilateral tinnitus in left and right ears, respectively. The mean tinnitus duration was $1.85 \pm 0.78$ years. As expected, there was not significant difference in terms of age $(P=0.88)$, employment duration $(P=0.83)$, workplace noise level $(P=0.78)$, and duration of tinnitus $(P=0.62)$ between two randomized study groups.

Participants were categorized based on the level of experienced changes in the severity of tinnitus quantified by VAS 
TABLE 1: Comparison of changes in tinnitus visual analog scaling and tinnitus handicap inventory scores immediately and 3 months after intervention between groups.

\begin{tabular}{|c|c|c|c|c|c|c|}
\hline \multirow{2}{*}{ Variable [number (\%)] } & \multicolumn{3}{|c|}{ Immediately after intervention } & \multicolumn{3}{|c|}{3 months after intervention } \\
\hline & Laser therapy group & Placebo group & $P$ value & Laser therapy group & Placebo group & $P$ value \\
\hline \multicolumn{7}{|l|}{ Visual analog scale score } \\
\hline No difference & $22(54)$ & $35(85)$ & & $29(70)$ & $40(97)$ & \\
\hline$<50 \%$ reduction & $7(17)$ & $3(7.5)$ & 0.006 & $5(13)$ & $1(3)$ & 0.003 \\
\hline$\geq 50 \%$ reduction & $12(29)$ & $3(7.5)$ & & $7(17)$ & $0(0)$ & \\
\hline \multicolumn{7}{|c|}{ Tinnitus handicap inventory score } \\
\hline No difference & $21(51)$ & $36(87)$ & & $27(66)$ & $40(97)$ & \\
\hline$<50 \%$ reduction & $2(6)$ & $1(3)$ & 0.001 & $1(3)$ & $0(0)$ & 0.001 \\
\hline$\geq 50 \%$ reduction & $18(43)$ & $4(10)$ & & $13(31)$ & $1(3)$ & \\
\hline
\end{tabular}

TABLE 2: Comparison of tinnitus loudness between two groups at baseline, immediately and 3 months after intervention.

\begin{tabular}{lccc}
\hline Tinnitus loudness $(\mathrm{dB})$ & Placebo & Laser therapy group & $P$ value \\
\hline Baseline & $6.09 \pm 1.11$ & $6.07 \pm 1.12$ & 0.922 \\
Immediately after intervention & $5.97 \pm 1.03$ & $4.51 \pm 1.89$ & $<0.001$ \\
3 months after intervention & $6.02 \pm 1.15$ & $5.09 \pm 1.90$ & 0.009 \\
\hline
\end{tabular}

${ }^{*}$ Data are presented as mean \pm standard deviation.

TABLE 3: Comparison of the changes of tinnitus loudness in 3 periods of assessment within and between groups.

\begin{tabular}{|c|c|c|c|c|c|}
\hline \multirow{2}{*}{ Tinnitus loudness $(\mathrm{dB})$} & \multicolumn{2}{|c|}{ Placebo } & \multicolumn{2}{|c|}{ Laser } & \multirow{2}{*}{$P$ value for between-group comparison } \\
\hline & Mean \pm SD & $P$ value & Mean \pm SD & $P$ value & \\
\hline Baseline & $6.09 \pm 1.11$ & \multirow{2}{*}{0.023} & $6.07 \pm 1.11$ & \multirow{2}{*}{$<0.001$} & \multirow{2}{*}{$<0.001$} \\
\hline Immediately after intervention & $5.97 \pm 1.03$ & & $4.51 \pm 1.03$ & & \\
\hline Baseline & $6.09 \pm 1.11$ & \multirow{2}{*}{0.183} & $6.07 \pm 1.11$ & \multirow{2}{*}{$<0.001$} & \multirow{2}{*}{$<0.001$} \\
\hline 3 months after intervention & $6.02 \pm 1.15$ & & $5.09 \pm 1.15$ & & \\
\hline Immediately after intervention & $5.97 \pm 1.03$ & \multirow{2}{*}{0.421} & $4.51 \pm 1.03$ & \multirow{2}{*}{0.013} & \multirow{2}{*}{0.01} \\
\hline 3 months after intervention & $6.02 \pm 1.15$ & & $5.09 \pm 1.15$ & & \\
\hline
\end{tabular}

and total THI. Table 1 summarizes the results of betweengroup analyses of distribution of changes in different time intervals. As shown, LLLB was significantly more effective than placebo immediately and 3 months after treatment, which points to the efficacy of the study intervention. Nevertheless, tinnitus severity remained unchanged in 54\% and $70 \%$ of patients immediately and 3 months after receiving LLLB (as measured by VAS).

According to Table 2, tinnitus loudness scores were comparable between two groups at baseline. After receiving LLLB, tinnitus loudness score was diminished in a U-shape manner with significantly lower scores than placebo in all time points.

Changes in tinnitus loudness score were compared within and between groups in different time periods. As expressed in Table 3, LLLB reduced the loudness of tinnitus significantly in relation to the baseline values and compared with the placebo group in all time periods. A meaningful response was also detected in placebo-assigned individuals immediately after treatment, which still was significantly lower than that of the intervention group.

\section{Discussion}

Previously published studies have reported the efficacy of LLLT in decreasing tinnitus to be between 15-67\% [11]. Quantifying by VAS, our positive findings have been multiplied by some $[2,11,13]$, while negated by other studies $[15,21]$. Tauber et al. used 10 sessions of LLLT with two different wavelengths (635 and $839 \mathrm{~nm}$ ) during two weeks which was different from our practice [11]. Okhovat et al. were treated patients with 20 minute sessions a day for 20 days using the same wavelengths to our study [2]. The most similar protocol to ours was used by Yildirim et al., with considerable improvements which sustained after two months [13].

Mixed results were also obtained by studies that have used tinnitus loudness score as their primary outcome measure. While this study in line with Tauber et al. [11], Gungor et al. [22], Newman et al. [20], and Shiomi et al. [23] has found tinnitus loudness to be improved after LLLT, two evaluations have failed to show the same efficacy $[15,16]$. A noteworthy point to consider is the pronounced improvement reported by our patients after receiving placebo, which vanished at the 


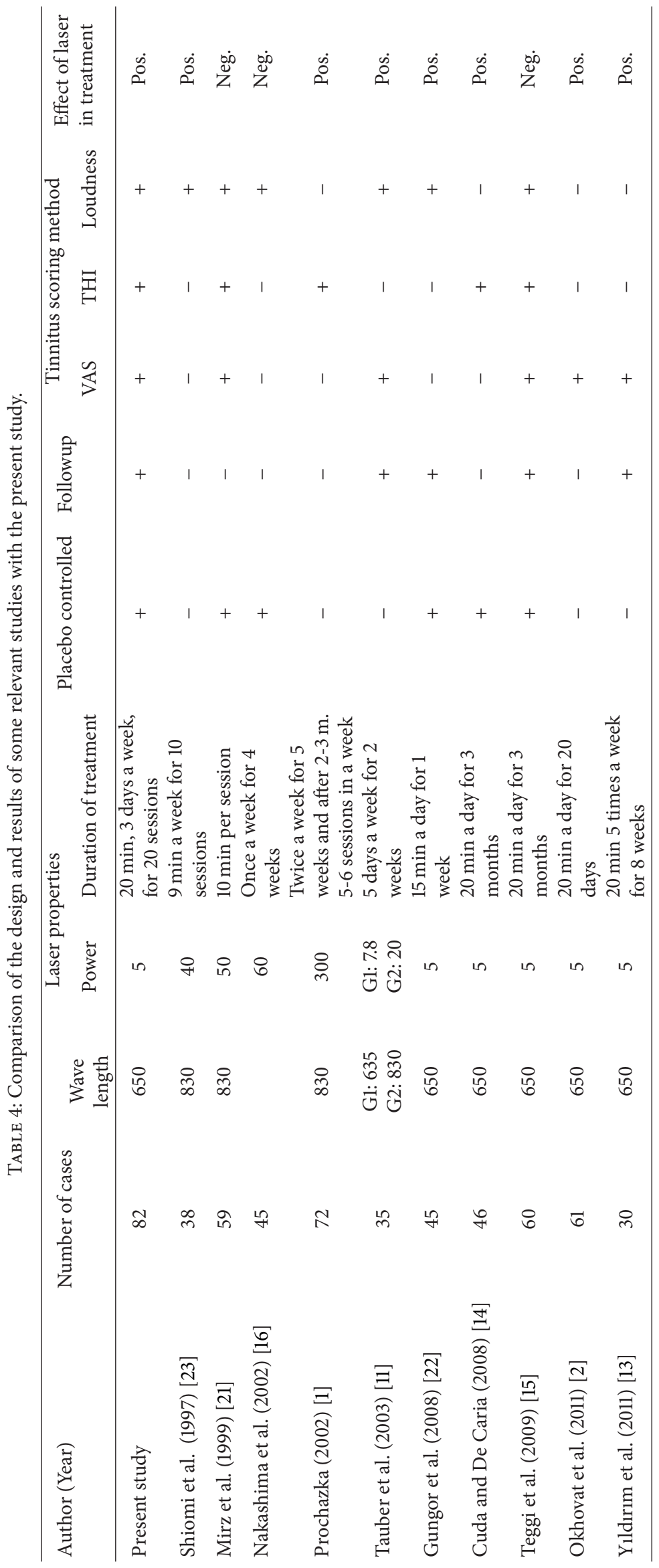


end of the follow-up period. This observation, to our opinion, might be explained best by a placebo effect.

The results of total THI score in our study were in accordance with VAS results after LLLT and were consistent with the study of Cuda and Caria [14], but Teggi et al. did not show this change [15]. Table 4 presents a detailed comparison between the findings of some relevant studies with what we found in our population. We suppose that the controversial results could be attributable to employing different treatment courses, as well as varied experiment settings. For instance, our patients received therapy in clinic, while Teggi et al. [15] gave the participants their course of treatment at home.

Even though it remained higher compared with the baseline level and placebo group, we observed that the effect of LLLT attenuated after 3 months. Our finding was attenuated by another research with assessment period of 4 weeks and 6 months [11]. It seems that the efficacy of LLLT decreases over time, which may necessitates repeating the therapy. Further evidence, however, is needed for determining a proper time interval between sessions.

While most of the former comparable studies have not taken concomitant hearing disorders into consideration, we assessed the effect of LLLT on tinnitus in a background of sensorineural hearing loss. However, our results should be interpreted in the light of some limitations. The first limitation was our 3-month follow-up period that made it impossible to evaluate long-term outcomes of the studied intervention. Secondly, due to the fact that our study population comprised of male workers, the obtained results may hardly be generalized to other populations.

In conclusion, this study has provided evidence for the efficacy of LLLT in relieving NIHL accompanied tinnitus, an effect that was weakened after 3 months follow-up. Despite significant improving results, the LLLB treatment nonresponse rate was considerable which should be taken into account when considering this treatment method.

\section{Conflict of Interests}

The authors declare that there is no conflict of interests that would prejudice the impartiality of this work.

\section{Acknowledgment}

The authors would like to appreciate the cooperation of all the personnel of Shahid Rahnemoun hospital and Industrial Related Diseases Research Center, Yazd, Iran. This study was financially supported by a grant from Shahid Sadoughi University of Medical Sciences (Grant no. 1563).

\section{References}

[1] M. Prochazka, "The Role of LLLT in Treatment of Tinnitus," 2002, http://ammhealth.co.za/pdf/info/latest/The\%20Role\%20 of $\% 20 L L L T \% 20$ in $\% 20$ Treatment $\% 20$ of $\% 20$ Tinnitus.pdf.

[2] A. Okhovat, N. Berjis, H. Okhovat, A. Malekpour, and H. Abtahi, "Low-level laser for treatment of tinnitus: a self-controlled clinical trial," Journal of Research in Medical Sciences, vol. 16, no. 1, pp. 33-38, 2011.
[3] A. Axelsson and A. Ringdahl, "Tinnitus-a study of its prevalence and characteristics," British Journal of Audiology, vol. 23, no. 1, pp. 53-62, 1989.

[4] A. H. Mehrparvar, S. J. Mirmohammadi, A. Ghoreyshi, A. Mollasadeghi, and Z. Loukzadeh, "High-frequency audiometry: a means for early diagnosis of noise-induced hearing loss," Noise and Health, vol. 13, no. 55, pp. 402-406, 2011.

[5] D. E. Dunn and P. M. Rabinowitz, "Noise," in Textbook of Clinical Occupational and Environmental Medicine, L. Rosenstock, Ed., p. 431, Elsevier Saunders, St. Louis, Mo, USA, 2nd edition, 2005.

[6] R. S. Tyler and L. J. Baker, "Difficulties experienced by tinnitus sufferers," Journal of Speech and Hearing Disorders, vol. 48, no. 2, pp. 150-154, 1983.

[7] P. Enrico, D. Sirca, and M. Mereu, "Antioxidants, minerals, vitamins, and herbal remedies in tinnitus therapy," Progress in Brain Research, vol. 166, pp. 323-330, 2007.

[8] B. Langguth, T. Kleinjung, M. Landgrebe, D. de Ridder, and G. Hajak, "rTMS for the treatment of tinnitus: the role of neuronavigation for coil positioning," Neurophysiologie Clinique, vol. 40, no. 1, pp. 45-58, 2010.

[9] O. Kapkin, B. Satar, and S. Yetiser, "Transcutaneous electrical stimulation of subjective tinnitus: a placebo-controlled, randomized and comparative analysis," ORL, vol. 70, no. 3, pp. 156$161,2008$.

[10] M. M. Jastreboff, "Sound therapies for tinnitus management," Progress in Brain Research, vol. 166, pp. 435-440, 2007.

[11] S. Tauber, W. Beyer, K. Schorn, and R. Baumgartner, "Transmeatal cochlear laser (TCL) treatment of cochlear dysfunction: a feasibility study for chronic tinnitus," Lasers in Medical Science, vol. 18, no. 3, pp. 154-161, 2003.

[12] L. Wilden, "The different response of the main symptoms of inner ear exhaustion to a specific high-dosage low-level laser therapy," Medical Laser Application, vol. 24, p. 133, 2009.

[13] G. Yıldırım, G. Berkiten, H. Uğraş, and Z. Saltürk, "Changes in audiometry results following laser therapy for tinnitus," European Journal of General Medicine, vol. 8, no. 4, pp. 284-290, 2011.

[14] D. Cuda and A. De Caria, "Effectiveness of combined counseling and low-level laser stimulation in the treatment of disturbing chronic tinnitus," International Tinnitus Journal, vol. 14, no. 2, pp. 175-180, 2008.

[15] R. Teggi, C. Bellini, L. O. Piccioni, F. Palonta, and M. Bussi, "Transmeatal low-level laser therapy for chronic tinnitus with cochlear dysfunction," Audiology and Neurotology, vol. 14, no. 2, pp. 115-120, 2009.

[16] T. Nakashima, H. Ueda, H. Misawa et al., "Transmeatal lowpower laser irradiation for tinnitus," Otology and Neurotology, vol. 23, no. 3, pp. 296-300, 2002.

[17] T. Moshkovska and J. Mayberry, "It is time to test low level laser therapy in Great Britain," Postgraduate Medical Journal, vol. 81, no. 957, pp. 436-441, 2005.

[18] American National Standards Institute, Specifications For Audiometers. ANSI S3. 6-2004, American National Standard Institute, Inc, New York, NY, USA, 2004.

[19] E. C. Huskisson, "Visual analogue scales," in Pain Measurement and Assessment, R. Melzack, Ed., pp. 33-37, Raven Press, New York, NY, USA, 1983.

[20] C. W. Newman, G. P. Jacobson, and J. B. Spitzer, "Development of the tinnitus handicap inventory," Archives of Otolaryngology, vol. 122, no. 2, pp. 143-148, 1996. 
[21] F. Mirz, R. Zachariae, S. E. Andersen et al., "The low-power laser in the treatment of tinnitus," Clinical Otolaryngology and Allied Sciences, vol. 24, no. 4, pp. 346-354, 1999.

[22] A. Gungor, S. Dogru, H. Cincik, E. Erkul, and E. Poyrazoglu, "Effectiveness of transmeatal low power laser irradiation for chronic tinnitus," Journal of Laryngology and Otology, vol. 122, no. 5, pp. 447-451, 2008.

[23] Y. Shiomi, H. Takahashi, I. Honjo, H. Kojima, Y. Naito, and N. Fujiki, "Efficacy of transmeatal low power laser irradiation on tinnitus: a preliminary report," Auris Nasus Larynx, vol. 24, no. 1, pp. 39-42, 1997. 


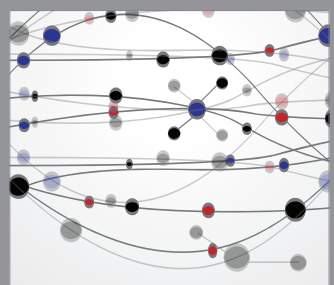

The Scientific World Journal
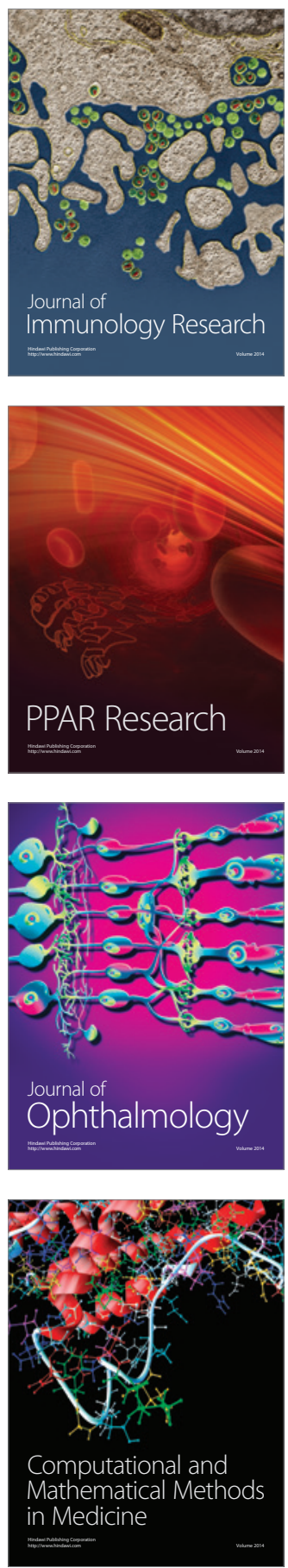

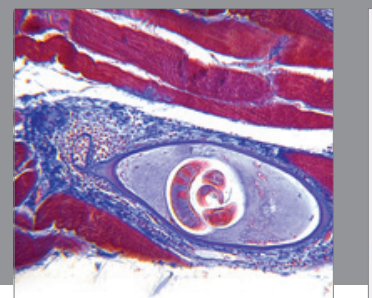

Gastroenterology

Research and Practice
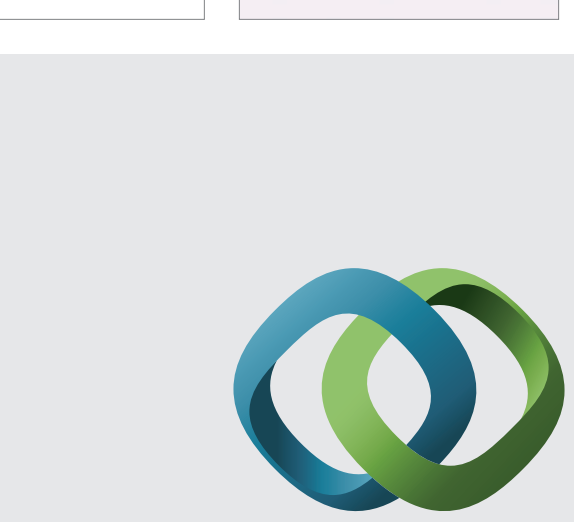

\section{Hindawi}

Submit your manuscripts at

http://www.hindawi.com
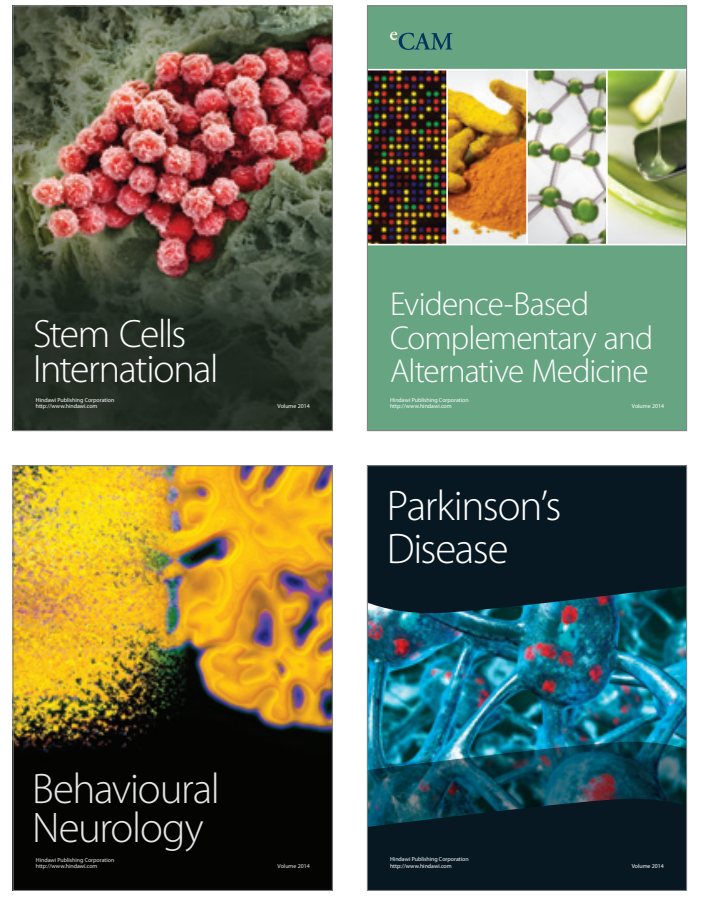
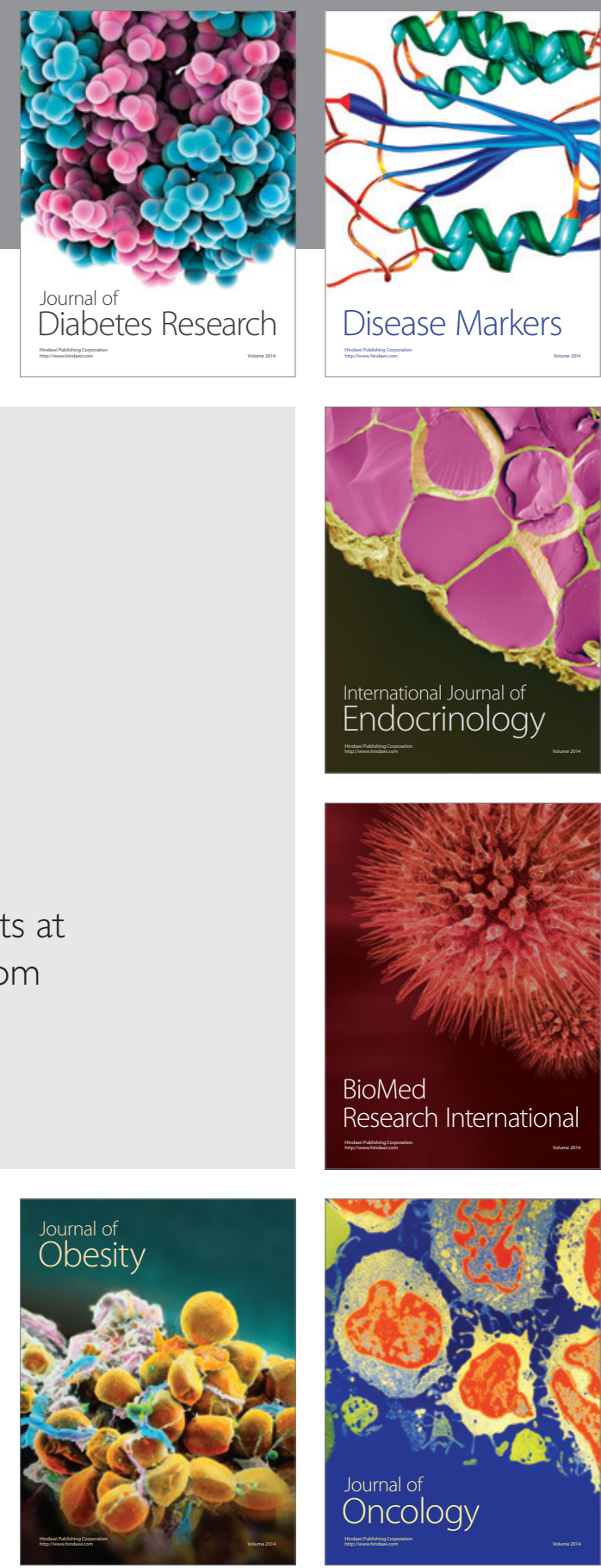

Disease Markers
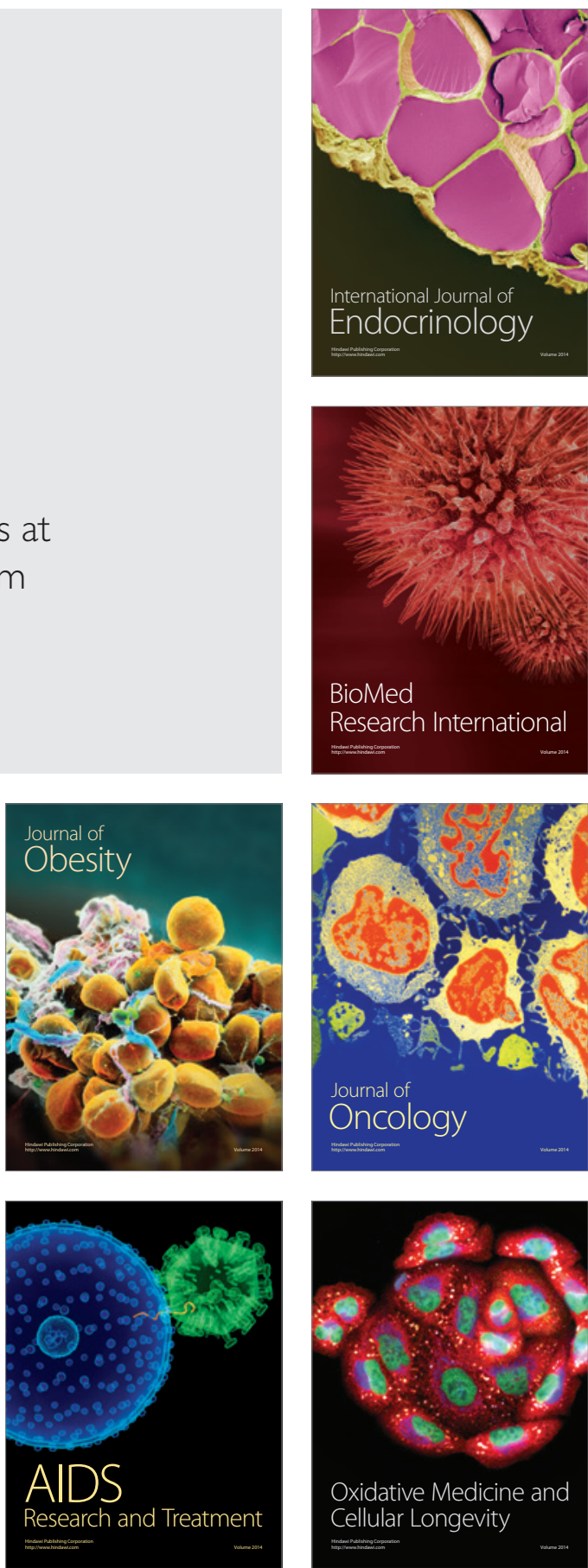